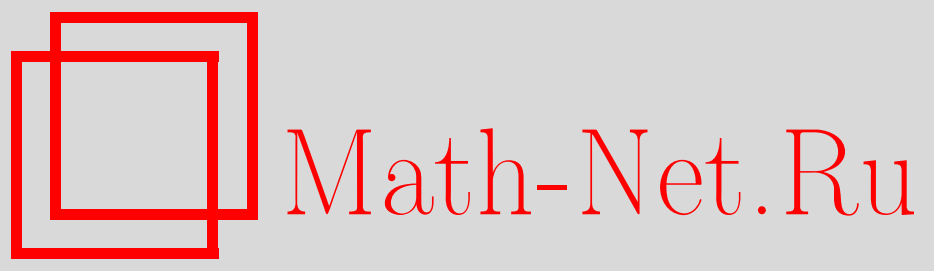

Gautami Bhowmik, Kohji Matsumoto, Analytic Continuation of Random Dirichlet Series, Совр. пробл. матем., 2013, выпуск 17, 76-81

DOI: https://doi.org/10.4213/spm44

Использование Общероссийского математического портала Math-Net.Ru подразумевает, что вы прочитали и согласны с пользовательским соглашением http://www.mathnet.ru/rus/agreement

Параметры загрузки:

IP: 54.210 .77 .194

26 апреля 2023 г., $17: 12: 59$

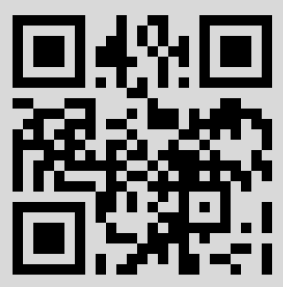




\title{
Analytic Continuation of Random Dirichlet Series
}

\author{
Gautami Bhowmik ${ }^{a}$, Kohji Matsumoto ${ }^{b}$ \\ ${ }^{a}$ Laboratoire Paul Painlevé, Université Lille 1, France \\ ${ }^{b}$ Graduate School of Mathematics, Nagoya University, Japan
}

Dedicated to the memory of Professor A. A. Karatsuba

\section{Introduction}

While studying analytic properties of Dirichlet series it is natural to ask whether and how far they can be meromorphically continued beyond their region of convergence. In general this is a difficult question, but when an answer can be found it usually corresponds to the intuitive natural boundary. The consideration of random Dirichlet series that can be shown to have natural boundaries almost surely comforts us in our belief that Dirichlet series should be continuable to their expected domains.

Random Dirichlet series and their convergence are differently treated depending on the context. In this note we first give an overview of existing results on natural boundaries. For the most part the series considered are in one variable. We suggest, in the second part of our note, a multiple analogue of random Dirichlet series. We indicate only some initial tools whose applications enable us to compare the abscissa of convergence of the Goldbach generating function with its random version. A more detailed study of these multiple analogues might turn out to be interesting.

\section{One variable}

We let $(\Omega, A, \mathrm{P})$ or simply $\Omega$ be a probability space and we say that an event $E$ in $A$ happens almost surely if $\mathrm{P}(E)=1$. Random Dirichlet series are of the form $\sum_{n=1}^{\infty} X_{n}(\omega) e^{-\lambda_{n} s}$, where the $\lambda_{i}$ form an increasing sequence of positive numbers, $X_{n}(\omega)$ are independent complex random variables in $(\Omega, A, \mathrm{P})$ and $s=\sigma+i t$ a complex number. A sequence of independent random variables $\varepsilon_{n}$ of $\Omega$ each of which takes only the values \pm 1 with the same probability $1 / 2$ is called a Rademacher sequence.

Relations between the different abscissae of convergence of random Dirichlet series have been studied since long. For example Hartman in 1931 considered Dirichlet series of the form $\sum_{n=1}^{\infty}\left( \pm a_{n}\right) n^{-s}$. There exist numbers $\sigma_{c} \leqslant \sigma_{u} \leqslant \sigma_{a}$ which are almost surely the abscissae of convergence, uniform convergence and absolute convergence respectively of such Dirichlet series. Hartman showed that $\sigma_{a}-\sigma_{u}$ is atmost $1 / 2$ and can be exactly $1 / 2$ [1]. The Bohr-Toeplitz theorem says that the same difference exists between the abscissae of absolute and uniform convergence for the ordinary Dirichlet series $\sum_{n=1}^{\infty} a_{n} n^{-s}$.

Dvoretzky in 1945 showed that for a given ordinary Dirichlet series $\sum_{n=1}^{\infty} a_{n} e^{-\lambda_{n} s}$, and for any $\sigma$ squeezed between its abscissae of convergence and absolute convergence one can always construct a random Dirichlet series $\sum_{n=1}^{\infty} \varepsilon_{n} a_{n} e^{-\lambda_{n} s}$ with $\operatorname{Re} s=\sigma$ as its natural boundary by an appropriate choice of $\varepsilon_{n}= \pm 1$ [2]. This is the analogue of the Fatou-Pólya theorem for power series.

New developments in the theory of random Dirichlet series began in 1970s. Yu (1978) studied particular cases where $\lambda_{n}$ satisfy the conditions $\lim _{n \rightarrow \infty}\left(\log n / \lambda_{n}\right)=0$ to obtain analogues of results on the radius of almost sure convergence for random power series [3].

Where the random variables are symmetric (as for example the case of Rademacher variables) the abscissa of convergence coincides almost surely with the natural boundary. Kahane described 
a dichotomy for the general case. The random series $\sum_{n=1}^{\infty} X_{n} e^{-\lambda_{n} s}$ either has the abscissa of convergence as its natural boundary or there exists an ordinary Dirichlet series $\sum_{n=1}^{\infty} a_{n} e^{-\lambda_{n} s}$ with the same abscissa of convergence $\sigma_{c}$ and a number $\sigma<\sigma_{c}$ such that the random series $\sum_{n=1}^{\infty}\left(X_{n}-a_{n}\right) e^{-\lambda_{n} s}$ has $\sigma$ as its natural boundary almost surely [4]. For the special series $\sum_{n=1}^{\infty} \pm n^{-s}$ he showed that almost surely the abscissa of convergence $\sigma_{c}=1 / 2$ would be its natural boundary [5]. This is the analogue of the Ryll-Nardzewski theorem for natural boundaries of random Taylor series. Some generalisations can be found in [6], [7].

Ding and Xiao studied explicit natural boundaries for uniformly nondegenerate variables $X_{n}$, i.e. $\sup _{n \geqslant 0} \sup _{a \in \mathbb{C}} \mathrm{P}\left(X_{n}=a\right)<1$, which is equivalent to the condition that there exists a sequence of positive numbers $R_{n}$ such that $\sup _{n \geqslant 0} \sup _{a \in \mathbb{C}} \mathrm{P}\left(\left|X_{n}-a\right| \leqslant R_{n}\right)<1$. If the abscissa of convergence of $\sum_{n=1}^{\infty} X_{n} e^{-\lambda_{n} s}$ is the same as that for $\sum_{n=1}^{\infty} R_{n}^{2} e^{-2 \lambda_{n} s}$ then this abscissa would almost surely be the natural boundary of the first series [8].

Queffélec enlarged the study by considering Euler products. He showed that $\prod\left(1-\varepsilon_{n} p_{n}^{-s}\right)^{-1}$, for $p_{n}$ the $n$th prime and $\left(\varepsilon_{n}\right)$ a sequence of independent Rademacher variables, has $\sigma=1 / 2$ almost surely as the natural boundary [9].

In the study of Euler products in both $p$ and $p^{-s}$ (for prime $p$ ), random variables were used in the exponents as an adaptation of the classical case. It was proved that the series $\prod_{n=1}^{\infty} \zeta\left(a_{n} s+b_{n}\right)^{c_{n}+\varepsilon_{n}}$, where $a_{n}, b_{n}, c_{n}$ are increasing real sequences and $\varepsilon_{n}$ a suitable sequence of independent real random variables, admits almost surely $\sigma=\lim _{\sup _{n \rightarrow \infty}}\left(-b_{n} / a_{n}\right)$ as its natural boundary [10].

Convergence of Random Dirichlet polynomials, finite versions of the Dirichlet series, have been studied, for example in [11].

\section{Several variables}

Now we suggest a multiple analogue of Rademacher sequences and associated Dirichlet series. Let $m_{1}, \ldots, m_{r} \in \mathbb{N}$, and let $\varepsilon_{m_{1}, \ldots, m_{r}}$ be independent random variables which take only the values \pm 1 and are defined on a certain probability space $(\Omega, A, \mathrm{P})$. Let

$$
\mathrm{P}\left(\omega \in \Omega \mid \varepsilon_{m_{1}, \ldots, m_{r}}(\omega)=1\right)=\mathrm{P}\left(\omega \in \Omega \mid \varepsilon_{m_{1}, \ldots, m_{r}}(\omega)=-1\right)=\frac{1}{2} .
$$

The sequence $\left\{\varepsilon_{m_{1}, \ldots, m_{r}} \mid m_{1}, \ldots, m_{r} \in \mathbb{N}\right\}$ may be called a Rademacher sequence of $r$-tuple indices. The associated multiple Dirichlet series can be defined as

$$
F_{r}(s, \omega)=\sum_{m_{1}=1}^{\infty} \cdots \sum_{m_{r}=1}^{\infty} \frac{\varepsilon_{m_{1}, \ldots, m_{r}}(\omega) X\left(m_{1}, \ldots, m_{r}\right)}{\left(m_{1}+\cdots+m_{r}\right)^{s}},
$$

where $s \in \mathbb{C}$ and $X\left(m_{1}, \ldots, m_{r}\right) \in \mathbb{R}$.

We note that other definitions of multiple random Dirichlet series exist (see, for example, [12] or [13]).

First we write $F_{r}(s, \omega)$ in a form of a single Dirichlet series:

$$
F_{r}(s, \omega)=\sum_{n=1}^{\infty} Y_{r}(n, \omega) n^{-s}
$$

where

$$
Y_{r}(n, \omega)=\sum_{m_{1}+\cdots+m_{r}=n} \varepsilon_{m_{1}, \ldots, m_{r}}(\omega) X\left(m_{1}, \ldots, m_{r}\right) .
$$

Since $\varepsilon_{m_{1}, \ldots, m_{r}}$ are independent variables, we see that $Y_{r}(n, \omega)$ are also independent of each other.

For each fixed $\omega \in \Omega$, the series (3.3) is a Dirichlet series, so we can find its abscissa of convergence $\sigma_{c}\left(F_{r}, \omega\right)$. Then, according to the zero-one law, we can find a constant $\sigma_{c}\left(F_{r}\right)$ such that $\sigma_{c}\left(F_{r}, \omega\right)=$ 
$\sigma_{c}\left(F_{r}\right)$ almost surely (see [4; Sect. 4.6]). In general $-\infty \leqslant \sigma_{c}\left(F_{r}\right) \leqslant \infty$, but here we assume $-\infty<\sigma_{c}\left(F_{r}\right)<\infty$. The following result is obtained simply.

Proposition 1. The line $\operatorname{Re} s=\sigma_{c}\left(F_{r}\right)$ is the natural boundary of $F_{r}(s, \omega)$ almost surely.

Proof. We say that $Y_{r}(n, \omega)$ is symmetric if $Y_{r}(n, \omega)$ and $-Y_{r}(n, \omega)$ have the same distribution. We prove that $Y_{r}(n, \omega)$ is symmetric; and the conclusion then follows immediately from the expression (3.3) and [4; Theorem 4, Sect. 4.6].

Let $L(n)$ be the number of tuples $\left(m_{1}, \ldots, m_{r}\right)$ satisfying $m_{1}+\cdots+m_{r}=n$. Let $\mathbf{b}$ be any tuple of $L(n)$ elements, each element being 1 or -1 . Write $\mathbf{b}=\left(b_{m_{1}, \ldots, m_{r}}\right)_{m_{1}+\cdots+m_{r}=n}$, where $b_{m_{1}, \ldots, m_{r}} \in\{ \pm 1\}$. Define

$$
Z_{r}(n, \mathbf{b})=\sum_{m_{1}+\cdots+m_{r}=n} b_{m_{1}, \ldots, m_{r}} X\left(m_{1}, \ldots, m_{r}\right) .
$$

Let $A$ be any Borel subset of $\mathbb{R}$, and let $\mathcal{B}(A)$ be the set of all $\mathbf{b}$ such that $Z_{r}(n, \mathbf{b}) \in A$. Then

$$
\mathrm{P}\left(\omega \in \Omega \mid Y_{r}(n, \omega) \in A\right)=\sum_{\mathbf{b} \in \mathcal{B}(A)} \mathrm{P}\left(\omega \in \Omega \mid\left(\varepsilon_{m_{1}, \ldots, m_{r}}(\omega)\right)_{m_{1}+\cdots+m_{r}=n}=\mathbf{b}\right) .
$$

Since $\varepsilon_{m_{1}, \ldots, m_{r}}$ are independent, we have

$$
\mathrm{P}\left(\omega \in \Omega \mid\left(\varepsilon_{m_{1}, \ldots, m_{r}}(\omega)\right)_{m_{1}+\cdots+m_{r}=n}=\mathbf{b}\right)=2^{-L(n)},
$$

and the same equality holds if we replace $\mathbf{b}$ by $-\mathbf{b}$. Therefore the right-hand side of (3.5) is equal to

$$
\sum_{\mathbf{b} \in \mathcal{B}(A)} \mathrm{P}\left(\omega \in \Omega \mid\left(\varepsilon_{m_{1}, \ldots, m_{r}}(\omega)\right)_{m_{1}+\cdots+m_{r}=n}=-\mathbf{b}\right)=\mathrm{P}\left(\omega \in \Omega \mid Y_{r}(n, \omega) \in-A\right) .
$$

This implies that two random variables $Y_{r}(n, \omega)$ and $-Y_{r}(n, \omega)$ have the same distribution.

\section{An example: The Goldbach generating functions}

As an example, we will treat the case where $X\left(m_{1}, \ldots, m_{r}\right)$ is a product of the von Mangoldt functions, that is

$$
\Phi_{r}(s, \omega)=\sum_{m_{1}=1}^{\infty} \cdots \sum_{m_{r}=1}^{\infty} \frac{\varepsilon_{m_{1}, \ldots, m_{r}}(\omega) \Lambda\left(m_{1}\right) \cdots \Lambda\left(m_{r}\right)}{\left(m_{1}+\cdots+m_{r}\right)^{s}} .
$$

The ordinary multiple Dirichlet series

$$
\Phi_{r}(s)=\sum_{m_{1}=1}^{\infty} \cdots \sum_{m_{r}=1}^{\infty} \frac{\Lambda\left(m_{1}\right) \cdots \Lambda\left(m_{r}\right)}{\left(m_{1}+\cdots+m_{r}\right)^{s}}
$$

was introduced in [14] as the generating function of

$$
G_{r}(n)=\sum_{m_{1}+\cdots+m_{r}=n} \Lambda\left(m_{1}\right) \cdots \Lambda\left(m_{r}\right)
$$

the latter being connected to the problem of expressing positive integers as a sum of $r$ prime numbers. Properties of $\Phi_{r}$ and $G_{r}$ above have been studied actively in the recent past (see [15] for references). It may be interesting to note that under the assumption of the Riemann Hypothesis $(\mathrm{RH})$ for the Riemann zeta-function $\zeta(s)$ whose non-trivial zeros are denoted by $\rho$,

$$
\sum_{n \leqslant X} G_{k}(n)=\frac{1}{k !} X^{k}+H_{k}(X)+\mathcal{O}_{\varepsilon}\left(X^{r-1+\varepsilon}\right)
$$


with

$$
H_{k}(X)=\sum_{\rho} \frac{X^{k-1+\rho}}{\rho(1+\rho) \cdots(k-1+\rho)} .
$$

Concerning the question of convergence of $\Phi_{r}(s)$ it was conjectured in [14] that:

The line $\operatorname{Re} s=r-1$ would be the natural boundary of $\Phi_{r}(s)$.

The conjecture is out of reach for the moment. However one can say more under the $\mathrm{RH}$ and other reasonable ones on the zeros of the Riemann zeta function like the following due to Fujii [16] [17]:

Let $\mathcal{I}$ be the set of all imaginary parts of non-trivial zeros of $\zeta(s)$. If $\gamma_{j} \in \mathcal{I}$, $1 \leqslant j \leqslant 4$, and $\gamma_{1}+\gamma_{2}=\gamma_{3}+\gamma_{4} \neq 0$, then $\left\{\gamma_{1}, \gamma_{2}\right\}=\left\{\gamma_{3}, \gamma_{4}\right\}$.

In [14] and [18], it was shown that (C-2) is indeed true under the $\mathrm{RH}$ and a certain quantitative version of $(\mathrm{Z})$ or $(\mathrm{Z})$ itself. The case of $(\mathrm{C}-r)$ is shown to be true if and only if $(\mathrm{C}-2)$ is.

The reason of introducing the random series (4.1) is to observe the situation from a stochastic viewpoint. Let

$$
G_{r}(n, \omega)=\sum_{m_{1}+\cdots+m_{r}=n} \varepsilon_{m_{1}, \ldots, m_{r}}(\omega) \Lambda\left(m_{1}\right) \cdots \Lambda\left(m_{r}\right) .
$$

Then

$$
\left|G_{r}(n, \omega)\right| \leqslant n^{r-1}(\log n)^{r}
$$

and hence (4.1), which can be written as

$$
\Phi_{r}(s, \omega)=\sum_{n=1}^{\infty} G_{r}(n, \omega) n^{-s}
$$

is absolutely convergent for $\operatorname{Re} s>r$. Computations of expectation and variance give us

PROPOSITION 2. The random series (4.7) converges for $\operatorname{Re} s>r / 2$ almost surely.

Proof. Let $\mathrm{E}(\cdot)$ denote the expected value, and $\mathrm{V}(\cdot)$ the variance. Write $X_{n}=G_{r}(n, \omega) n^{-s}$. From (4.6) we see that

$$
\int_{\Omega}\left|X_{n}\right|^{2} d \omega \leqslant\left(\frac{n^{r-1}(\log n)^{r}}{n^{\sigma}}\right)^{2} \int_{\Omega} d \omega<+\infty
$$

that is $X_{n} \in L^{2}(\Omega)$.

We use Theorem 2 in [4; Sect. 3.2], which asserts that if $X_{n} \in L^{2}(\Omega)$ are independent random variables satisfying $\mathrm{E}\left(X_{n}\right)=0$ and $\sum_{n=1}^{\infty} \mathrm{V}\left(X_{n}\right)<+\infty$, then $\sum_{n=1}^{\infty} X_{n}$ converges almost surely.

Since

$$
\mathrm{E}\left(X_{n}\right)=\frac{1}{n^{s}} \sum_{m_{1}+\cdots+m_{r}=n} \Lambda\left(m_{1}\right) \cdots \Lambda\left(m_{r}\right) \int_{\Omega} \varepsilon_{m_{1}, \ldots, m_{r}}(\omega) d \omega,
$$

and $\varepsilon_{m_{1}, \ldots, m_{r}}$ is an element of a Rademacher sequence, it is obvious that $\mathrm{E}\left(X_{n}\right)=0$ for any $n$. Next consider $\mathrm{V}\left(X_{n}\right)=\mathrm{E}\left(\left|X_{n}-\mathrm{E}\left(X_{n}\right)\right|^{2}\right)=\mathrm{E}\left(\left|X_{n}\right|^{2}\right)$. We see that

$$
\mathrm{V}\left(X_{n}\right)=\frac{1}{n^{2 \sigma}} \sum_{\substack{m_{1}+\cdots+m_{r}=n \\ m_{1}^{\prime}+\cdots+m_{r}^{\prime}=n}} \Lambda\left(m_{1}\right) \cdots \Lambda\left(m_{r}\right) \Lambda\left(m_{1}^{\prime}\right) \cdots \Lambda\left(m_{r}^{\prime}\right) \int_{\Omega} \varepsilon_{m_{1}, \ldots, m_{r}}(\omega) \varepsilon_{m_{1}^{\prime}, \ldots, m_{r}^{\prime}}(\omega) d \omega
$$


If $\left(m_{1}, \ldots, m_{r}\right) \neq\left(m_{1}^{\prime}, \ldots, m_{r}^{\prime}\right)$, then

$$
\mathrm{P}\left(\omega \in \Omega \mid \varepsilon_{m_{1}, \ldots, m_{r}}(\omega)= \pm 1, \quad \varepsilon_{m_{1}^{\prime}, \ldots, m_{r}^{\prime}}(\omega)= \pm 1\right)=\frac{1}{4}
$$

for any choice of double signs, hence

$$
\int_{\Omega} \varepsilon_{m_{1}, \ldots, m_{r}}(\omega) \varepsilon_{m_{1}^{\prime}, \ldots, m_{r}^{\prime}}(\omega) d \omega=0 .
$$

Therefore,

$$
\begin{aligned}
\mathrm{V}\left(X_{n}\right) & =\frac{1}{n^{2 \sigma}} \sum_{m_{1}+\cdots+m_{r}=n} \Lambda\left(m_{1}\right)^{2} \cdots \Lambda\left(m_{r}\right)^{2} \int_{\Omega} \varepsilon_{m_{1}, \ldots, m_{r}}(\omega)^{2} d \omega \\
& =\frac{1}{n^{2 \sigma}} \sum_{m_{1}+\cdots+m_{r}=n} \Lambda\left(m_{1}\right)^{2} \cdots \Lambda\left(m_{r}\right)^{2},
\end{aligned}
$$

and the sum on the right-hand side can be estimated as $\leqslant n^{r-1}(\log n)^{2 r}$. Therefore

$$
\sum_{n=1}^{\infty} \mathrm{V}\left(X_{n}\right) \leqslant \sum_{n=1}^{\infty} n^{r-1-2 \sigma}(\log n)^{2 r}
$$

which is convergent if $\sigma>r / 2$.

Proposition 2 thus gives the upper-bound $\sigma_{c}\left(\Phi_{r}\right) \leqslant r / 2$. We also comment on its lower-bound. Since $G_{r}$ is symmetric (as we have seen in the proof of Proposition 1), we have the criterion that $\sum_{n=1}^{\infty} X_{n}$ converges almost surely if and only if $\sum_{n=1}^{\infty} \mathrm{V}\left(X_{n}^{\prime}\right)$ converges, where

$$
X_{n}^{\prime}(\omega)= \begin{cases}X_{n}(\omega) & \text { if }\left|X_{n}(\omega)\right| \leqslant 1 \\ \frac{X_{n}(\omega)}{\left|X_{n}(\omega)\right|} & \text { if }\left|X_{n}(\omega)\right|>1\end{cases}
$$

([4; Theorem 7, Sect. 3.5]).

In the case $r=1$, we have $X_{n}(\omega)=\varepsilon_{n}(\omega) \Lambda(n) n^{-s}$. Hence if $\sigma>0$ then $\left|X_{n}(\omega)\right| \leqslant 1$ for sufficiently large $n$, so we may assume $X_{n}^{\prime}=X_{n}$ if $\sigma>0$. From (4.11) we have

$$
\sum_{n=1}^{\infty} \mathrm{V}\left(X_{n}\right)=\sum_{n=1}^{\infty} \frac{\Lambda(n)^{2}}{n^{2 \sigma}}
$$

which is convergent if and only if $\sigma>1 / 2$. This implies that $\sigma_{c}\left(\Phi_{1}\right)=1 / 2$, and hence, by Proposition 1 , the line $\operatorname{Re} s=1 / 2$ is the natural boundary of $\Phi_{1}(s, \omega)$ almost surely.

Next consider the case $r=2$. Then

$$
X_{n}(\omega)=\frac{1}{n^{s}} \sum_{m_{1}+m_{2}=n} \varepsilon_{m_{1}, m_{2}} \Lambda\left(m_{1}\right) \Lambda\left(m_{2}\right) .
$$

If $\sigma>1$ then we may assume $X_{n}^{\prime}=X_{n}$ for sufficiently large $n$. From (4.11) we have

$$
\sum_{n=1}^{\infty} \mathrm{V}\left(X_{n}\right)=\sum_{m_{1}+m_{2}=n} \frac{\Lambda\left(m_{1}\right)^{2} \Lambda\left(m_{2}\right)^{2}}{n^{2 \sigma}}
$$

whose right-hand side is

$$
\geqslant \frac{1}{n^{2 \sigma}} \sum_{\substack{p_{i}, p_{2} \text { prime } \\ p_{1}+p_{2}=n}}\left(\log p_{1}\right)^{2}\left(\log p_{2}\right)^{2} \gg \frac{(\log n)^{2}}{n^{2 \sigma}} \sum_{\substack{p_{i}, p_{2} \text { prime } \\ p_{1}+p_{2}=n}} 1,
$$


because one of $p_{1}$ or $p_{2}$ is $\geqslant n / 2$. If the Hardy-Littlewood conjectural asymptotic formula for the Goldbach conjecture is true, then the last sum of $(4.16)$ is $\gg n(\log n)^{-2}$. This implies that (4.15) diverges for $\sigma=1$. Though $\sigma=1$ is out of the range where $X_{n}^{\prime}=X_{n}$ is valid, this argument suggests that $\sigma_{c}\left(\Phi_{2}\right)=1$, and hence Re $s=1$ would be the natural boundary of $\Phi_{2}(s, \omega)$ almost surely. This is consistent with the existent conditional natural boundary of $\Phi_{2}(s)$.

The above observation in the cases $r=1$ and $r=2$ further suggests that in the case $r \geqslant 3$, perhaps $\sigma_{c}\left(\Phi_{r}\right)=r / 2$ would hold.

\section{Bibliography}

[1] P. Hartman, "On Dirichlet series involving random coefficients", Amer. J. Math., 61:4 (1939), 955-964.

[2] A. Dvoretzky, "Sur les changements des signes de coefficients des séries de Dirichlet", C. R. Acad. Sci. Paris, 221 (1945), 687-689.

[3] J. R. Yu, "Some properties of random Dirichlet series", Acta Math. Sinica, 21:2 (1978), 97-118.

[4] J.-P. Kahane, Some Random Series of Functions, Cambridge Stud. Adv. Math., 5, Cambridge Univ. Press, Cambridge, 1985.

[5] J.-P. Kahane, "Sur les séries de Dirichlet $\sum_{1}^{\infty} \pm n^{-s ", ~ C . ~ R . ~ A c a d . ~ S c i . ~ P a r i s ~ S e ́ r . ~ A, ~ 276: 4 ~(1973), ~}$ 739-742.

[6] P. V. Filevych, "On relations between the abscissa of convergence and the abscissa of absolute convergence of random Dirichlet series", Mat. Stud., 20:1 (2003), 33-39.

[7] F. Tian, D. Sun, J. Yu, "Sur les séries aléatoires de Dirichlet", C. R. Acad. Sci. Paris Sér. I Math., 326:4 (1998), 427-431.

[8] X. Ding, Y. Xiao, "Natural boundary of random Dirichlet series", Ukrainian Math. J., 58:7 (2006), 1129-1138.

[9] H. Queffélec, "Propriétés presque sûres et quasi-sûres des séries de Dirichlet et des produits d'Euler", Canad. J. Math., 32:3 (1980), 531-558.

[10] G. Bhowmik, J.-C. Schlage-Puchta, "Natural boundaries of Dirichlet series", Funct. Approx. Comment. Math., 37:1 (2007), 17-29.

[11] M. Lifshits, M. Weber, "On the supremum of some random Dirichlet polynomials", Acta Math. Hungar., 123:1-2 (2009), 41-64.

[12] O. B. Skaskiv, O. Yu. Zadorozhna, "On domains of convergence of multiple random Dirichlet series", Mat. Stud., 36:1 (2011), 51-57.

[13] F. Tian, "On the convergence of the double random Dirichlet series", J. Hubei Univ., Nat. Sci., 22:4 (2000), 317-320.

[14] S. Egami, K. Matsumoto, "Convolutions of the von Mangoldt function and related Dirichlet series", Number Theory. Sailing on the Sea of Number Theory, Ser. Number Theory Appl., 2, World Sci. Publ., Hackensack, NJ, 2007, 1-23.

[15] G. Bhowmik, "Analytic continuation of some zeta functions", Algebraic and Analytic Aspects of Zeta Functions and L-Functions, MSJ Mem., 21, Math. Soc. Japan, Tokyo, 2010, 1-16.

[16] A. Fujii, "An additive problem of prime numbers", Acta Arith., 58:2 (1991), 173-179.

[17] A. Fujii, "An additive problem of prime numbers. II", Proc. Japan Acad. Ser. A Math. Sci., 67:7 (1991), 248-252; "An additive problem of prime numbers. III", 67:8 (1991), 278-283.

[18] G. Bhowmik, J.-C. Schlage-Puchta, "Meromorphic continuation of the Goldbach generating function", Funct. Approx. Comment. Math., 45:1 (2011), 43-53. 